\title{
SOME SMALL ASPHERICAL SPACES
}

\author{
Dedicated to the memory of Hanna Neumann
}

\author{
ELDON DYER and A. T. VASQUEZ
}

(Received 26 June 1972)

Communicated by M. F. Newman

Let $S^{n}$ denote the sphere of all points in Euclidean space $\mathbf{R}^{n+1}$ at a distance of 1 from the origin and $D^{n+1}$ the ball of all points in $\mathbf{R}^{n+1}$ at a distance not exceeding 1 from the origin. The space $X$ is said to be aspherical if for every $n \geqq 2$ and every continuous mapping: $f: S^{n} \rightarrow X$, there exists a continuous mapping $g: D^{n+1} \rightarrow X$ with restriction to the subspace $S^{n}$ equal to $f$. Thus, the only homotopy group of $X$ which might be non-zero is the fundamental group $\pi_{1}\left(X,{ }^{*}\right) \cong G$. If $X$ is also a cell-complex, it is called a $K(G, 1)$. If $X$ and $Y$ are $K(G, 1)$ 's, then they have the same homotopy type, and consequently

$$
H_{i}(X ; M) \cong H_{i}(Y ; M) \text { and } H^{i}(X ; M) \cong H^{i}(Y ; M)
$$

for all coefficients $M$ and integers $i$. If $M$ is a left $G$-module, it determines a local coefficient system over $X$. The groups

$$
H_{i}(X ; M) \text { and } H^{i}(X ; M)
$$

are thus invariants of the group $G$ and module $M$. They are called the homology and cohomology groups of $G$ with coefficients $M$, written

$$
H_{i}(G ; M) \text { and } H^{i}(G ; M)
$$

(These observations, essentially due to H. Hopf, led to the development of homological algebra, which initiated the study of these groups by algebraic means.)

For a given discrete group $G$, it may be that there is an integer $n$ such that for all left $G$-modules $M$ and integers $i>n, H^{i}(G ; M)=0$. If so, then $G$ is said to be of finite cohomological dimension and the minimum of all such integers $n$ is defined to be the cohomological dimension of $G$, written $c d G$.

It might also be that there exists a $K(G, 1)$ which has finite topological dimension. If so, the minimum such dimension is called the geometric dimension of $G$, written $\operatorname{dim} G$. 
It is easy to show that $c d G \leqq \operatorname{dim} G$. In [3], Eilenberg and Ganea showed that when $3 \leqq c d G, c d G=\operatorname{dim} G$. In [11] and [13], Stallings and Swan showed if $1=c d G$, then $c d G=\operatorname{dim} G$. The case $2=c d G$ is unresolved at this time.

This paper presents explicit cellular constructions of 2-dimensional $K(G, 1)$ 's for certain families of groups $G$. The techniques involve elementary rewriting arguments and covering space constructions. This recovers and casts new light on the Lyndon Identity Theorem. Based on a group described by Higman [4], a 2-dimensional $K(G, 1)$ is given which has curious topological properties. Also, conjecture $I^{\prime}$ of Papakyriakopoulos [8] is answered affirmatively.

The authors take this opportunity to thank publicly Professor Joan Dyer for a number of conversations helpful in the preparation of this paper.

\section{Preliminaries}

We present several constructions and lemmas which will be used subsequently.

Let $X$ denote a pathwise connected space and $A$ denote a subspace of $X$ which is simply and pathwise connected. Each path $\beta$ in $X$ induces an isomorphism

$$
\beta_{*}: \pi_{1}(X, \beta(1)) \rightarrow \pi_{1}(X, \beta(0))
$$

given by $\beta_{*} \omega=\beta \omega \beta^{-1}$ for $\omega$ a loop at $\beta(1)$. The isomorphism $\beta_{*}$ depends only on the homotopy class of $\beta$ relative to its end points. Since $A$ is both pathwise connected and simply connected, for any two points $a_{0}$ and $a_{1}$ of $A$, there is a path $\alpha$ from $a_{0}$ to $a_{1}$ and any two such paths are homotopic relative to their end points. Thus, there is a well defined isomorphism

$$
\pi_{1}\left(X, a_{0}\right) \cong \pi_{1}\left(X, a_{1}\right)
$$

for any two points $a_{0}$ and $a_{1}$ of $A$. We define $\pi_{1}(X, A)$ to be the subgroup of the group $\prod_{a \in A} \pi_{1}(X, a)$ defined by $n \in \pi_{1}(X, A)$ if and only if for every two points $a_{0}$ and $a_{1}$ of $A, n\left(a_{0}\right) \in \pi_{1}\left(X, a_{0}\right)$ and $n\left(a_{1}\right) \in \pi_{1}\left(X, a_{1}\right)$ correspond under the above isomorphism. Clearly, for any $a \in A$ projection of $\pi_{1}(X, A)$ on the ath-coordinate is an isomorphism $\pi_{1}(X, A) \cong \pi_{1}(X, a)$. The advantage of $\pi_{1}(X, A)$ is that it is determined independently of base points.

LEMMA 1.1: Let $X$ be a pathwise connected, locally pathwise connected space, $A$ be a pathwise connected, simply connected subspace of $X$, and $H$ be a group of homeomorphisms of $X$ which acts properly discontinuously on $X$ and each of which transforms $A$ onto itself.

Then the fundamental group $G$ of the orbit space $X / H$ is isomorphic to the semi-direct product determined by the split extension

$$
1 \rightarrow N \rightarrow G \leftrightarrows H \rightarrow 1,
$$

where $N=\pi_{1}(X, A)$ and the action of $H$ on $N$ is the topologically induced action. 
Procf: The action of $H$ on $\pi_{1}(X, A)$ is defined by $h(n)(a)=\pi_{1}(h)\left(n\left(a^{\prime}\right)\right)$, where $h\left(a^{\prime}\right)=a$ and $\pi_{1}(h): \pi_{1}\left(X, a^{\prime}\right) \rightarrow \pi_{1}(X, a)$, for $h \in H$ and $n \in \pi_{1}(X, A)$.

Let $B=X / H$ be the space of orbits and $p: X \rightarrow B$ be the quotient map. It is well-known that $(X, p, B)$ is a regular covering space with $H$ being the group of convering transformations. Thus, for any $x_{0} \in X, b_{0}=p\left(x_{0}\right)$, there is an exact sequence

$$
1 \rightarrow \pi_{1}\left(X, x_{0}\right) \stackrel{p *}{\rightarrow} \pi_{1}\left(B, b_{0}\right) \stackrel{\rho}{\rightarrow} H \rightarrow 1 .
$$

Let $x_{0}=a_{0} \in A$. We shall show this sequence splits. For $h \in H$ and $\alpha$ a path in $A$ from $a_{0}$ to $h\left(a_{0}\right)$, set $s(h)=[p \alpha] \in \pi_{1}\left(B, b_{0}\right)$. This is well-defined, it is a homomorphism, and $\rho s=1_{H}$.

Thus, $\pi_{1}\left(B, b_{0}\right)$ is the semi-direct product of $\pi_{1}\left(X, a_{0}\right)$ and $H$ with $H$ acting on $\pi_{1}\left(X, a_{0}\right)$ defined by

$$
p_{*}(h(\gamma))=s(h) p_{*}(\gamma) s(h)^{-1} \in \pi_{1}\left(B, b_{0}\right) .
$$

Identifying $\pi_{1}\left(X, a_{0}\right)$ with $\pi_{1}(X, A)$ as above, we have left to show only that this action coincides with the induced action of $H$ on $\pi_{1}(X, A)$. Let $n \in \pi_{1}(X, A)$ and $h \in H$; let $\alpha$ be a path in $A$ from $a_{0}$ to $h\left(a_{0}\right)$ and $\beta$ be a loop at $a_{0}$ in the class of $n\left(a_{0}\right)$. We must show that

$$
s(h) p_{*}\left(n\left(a_{0}\right)\right) s(h)^{-1}=p_{*} h(n)\left(a_{0}\right) .
$$

The loop $p \alpha \cdot p \beta \cdot p \alpha^{-1}$ is in the homotopy class of $s(h) p_{*}\left(n\left(a_{0}\right)\right) s(h)^{-1}$ and $\alpha \cdot h \beta \cdot \alpha^{-1}$ is the lift of this loop stariing at $a_{0}$. Thus, $p_{*}\left(\alpha \cdot h \beta \cdot \alpha^{-1}\right)=s(h) p_{*}$ $\left(n\left(a_{0}\right)\right) s(h)^{-1}$. We need show only that $h(n)\left(a_{0}\right)=\left[\alpha \cdot h \beta \cdot \alpha^{-1}\right] \in \pi_{1}\left(X, a_{0}\right)$. Let $n^{\prime}$ be the element of $\pi_{1}(X, A)$ with $n^{\prime}\left(a_{0}\right)=\left[\alpha \cdot h \beta \cdot \alpha^{-1}\right]$. Since $\alpha$ lies in $A$, $n^{\prime}\left(a_{1}\right)=[h \beta]$, where $a_{1}=h\left(a_{0}\right)$. But $[h \beta]=\pi_{1}(h)[\beta]=\pi_{1}(h) n\left(a_{0}\right)=h(n)\left(a_{1}\right)$. Hence, $n^{\prime}\left(a_{1}\right)=h(n)\left(a_{1}\right)$; consequently, $n^{\prime}=h(n)$ and, in particular, they are equal at $a_{0}$.

Let $P:\left\{x_{1}, \cdots, x_{n} ; R_{1}, \cdots, R_{m}\right\}$ be a presentation of the discrete group $G$; i.e., $R_{1}, \cdots, R_{m}$ are words in the $x_{1}, \cdots, x_{n}$ and $G$ is the quotient of the free group $F\left(x_{1}, \cdots, x_{n}\right)$ on $x_{1}, \cdots, x_{n}$ by the normal closure in $F$ of the elements $R_{1}, \cdots, R_{m}$. The wedge $\bigvee_{i=1}^{n} S_{i}^{1}$ of $n 1$-spheres has fundamental group

$$
\pi_{1}\left(\bigvee_{i=1}^{n} S_{i}^{1}, *\right) \cong F\left(x_{1}, \cdots, x_{n}\right)
$$

with the generator $x_{\imath}$ corresponding to a map $S^{1} \rightarrow S_{\imath}^{1}$ of degree 1 . For each $j, 1$ $\leqq j \leqq m$, let $D_{j}^{2}$ be a 2-ball, $B_{j}^{1}$ be its boundary (which is a 1 -sphere) and

$$
f_{j}: B_{j}^{1} \rightarrow \bigvee_{i=1}^{n} S_{i}^{1}
$$

be a map such that if $t_{j}$ is a generator of $\pi_{1}\left(B_{j}^{1}\right) \cong Z$, then 


$$
f_{j_{*}}: \pi_{1}\left(B_{j}^{1}\right) \rightarrow \pi_{1}\left(\bigvee_{i=1}^{n} S_{i}^{1}\right)
$$

maps $t_{j}$ into the word $R_{j}$. Form the quotient space $C(P)$ from the disjoint union

$$
\bigvee_{i=1}^{n} S_{i}^{1} \cup \bigcup_{j=1}^{m} D_{j}^{2}
$$

by identifying $y \in B_{j}^{1}$ with $f_{j}(y)$ for every point in $\bigcup_{j=1}^{m} B_{j}^{\mathbf{1}}$. (This is sometimes described as attaching the cells $D_{j}^{2}$ by the maps $f_{j}$.) This quotient space is called the canonical cell-complex of the presentation $P$, written $C(P)$; it is a 2-dimensional cell-complex and an application of the Siefert-van Kampen Theorem shows

LEMMA 1.2: $I f$

$$
\pi_{1}\left(C(P),{ }^{*}\right) \cong G \text {. }
$$

and

$$
P:\left\{x_{1}, \cdots, x_{n} ; R_{1}, \cdots, R_{m}\right\}
$$

$$
P^{\prime}:\left\{x_{1}, \cdots, x_{n} ; \alpha_{1} R_{1}^{\varepsilon_{1}} \alpha_{1}^{-1}, \cdots, \alpha_{m} R_{m}^{\varepsilon_{m}} \alpha_{m}^{-1}\right\}, \varepsilon_{i}= \pm 1,
$$

are presentations, then $C(P)$ and $C\left(P^{\prime}\right)$ have the same unpointed homotopy type; one is aspherical if and only if the other is.

This is true basically because the attaching maps are freely homotopic.

We reca!l a theorem of J. H. C. Whitehead [14]:

TheOREM W: Suppose $X, Y$ and $X \cap Y$ are aspherical cell-complexes and both $\pi_{1}(X \cap Y, *) \rightarrow \pi_{1}(X, *)$ and $\pi_{1}(X \cap Y, *) \rightarrow \pi_{1}(Y, *)$ are injections. Then $X \cup Y$ is aspherical.

This theorem has a number of useful immediate consequences. Let $G, H$ and $A$ denote the fundamental groups of $X, Y$ and $X \cap Y$, respectively. Then by the Seifert-van Kampen Theorem, the fundamental group of $X \cup Y$ is the free product with amalgamation $G_{A}^{*} H$. Letting $M$ be a left $G * H$-module viewed as local coefficients over $X \cup Y$ and applying the Mayer-Vietoris Sequences for $(X, Y ; A)$, one obtains the Mayer-Vietoris Sequences for homology and cohomology of groups of free products with amalgamation: (This argument is essentially that of [10]; the result is indicated in [6] and a homological algebra proof appears in [13].)

It is immediate from these sequences that

$$
\max \{c d G, c d H\} \leqq c d G_{A}^{*} H \leqq 1+\max \{c d G, c d H\},
$$

with equality on the left applying if either $c d A<c d G$ or $c d A<c d H$. Using mapping cylinders for cellular maps $K(A, 1) \rightarrow K(G, 1)$ and $K(A, 1) \rightarrow K(H, 1)$ which induce the appropriate injections of fundamental groups, we can conclude from Whitehead's Theorem that 
$\max \{\operatorname{dim} G, \operatorname{dim} H\} \leqq \operatorname{dim} G_{A}^{*} H \leqq 1+\max \{\operatorname{dim} G, \operatorname{dim} H\}$

with equality on the left applying if either $\operatorname{dim} A<\operatorname{dim} G$ or $\operatorname{dim} A<\operatorname{dim} H$.

In particular, we have the generalized free product (free product amalgamated over a free group) of 2-dimensional groups is a 2-dimensional group. Of course, the same is true for cohomology dimension.

Using the homological algebra definition of cohomology of groups, since a projective resolution over a group remains projective over a sugroup, we have

if $H$ is a subgroup of $G, c d H \leqq c d G$.

Also, if $X$ is an $n$-dimensional $K(G, 1)$, the covering space of $X$ with fundamental group $H$ is an $n$-dimensional aspherical cell-complex. Thus,

if $H$ is a subgroup of $G, \operatorname{dim} H \leqq \operatorname{dim} G$.

In light of the Eilenberg-Ganea Theorem, this parallelism of conclusions is of interest only at 2 .

\section{One relator presentations}

Let $P:\left\{x_{1}, \cdots, x_{n} ; R\right\}$ be a one relator presentation for the group $G$. We assume $R$ is fully reduced, $R \neq 1$; if for some word $W$ in the $x_{i}$ 's and integer $t$, $R=w^{\prime}$, let $s$ be the maximal such integer and $Q$ the corresponding word, $R=Q^{s}$. If $s>1, R$ is said to be a proper power. Clearly, $Q$ is not a proper power.

For $s$ a positive integer, let $\mathbf{Z}_{s}$ denote the cyclic group of order $s, \mathbf{Z} / s \mathbf{Z}$. There is a cell-complex $K\left(Z_{s}, 1\right)$ with 1 -sphere $S^{1}$ as 1 -skeleton; for $s>1 K\left(Z_{s}, 1\right)$ can be formed with exactly one cell in each dimension (using infinite dimensional lens spaces \{p. 67 of [12]\}), and its homology and cohomology have been well understood for several decades. For $s=1, K(0,1)$ can be formed by adding the 2-cell $D^{2}$ to $S^{1}$ along its boundary. In any case the inclusion $S^{1} \rightarrow K\left(Z_{s}, 1\right)$ induces an epimorphism of fundamental groups $\mathbf{Z} \rightarrow \mathbf{Z}_{s}$. As in Section $\mathbf{I}$, let $f: S^{\mathbf{1}}$ $\rightarrow \bigvee_{i=1}^{n} S_{i}^{1}$ induce the homomorphism on fundamental groups sending a generator of $\pi_{1}\left(S^{1}\right)$ to the word $Q$ in $\pi_{1}\left(\bigvee_{i=1}^{n} S_{i}^{1}\right)$. From the disjoint union $K\left(Z_{s}, 1\right)$ $\cup \bigvee_{i=1}^{n} S_{i}^{1}$ form the quotient space $D(P)$ by identifying each $x \in S^{1} \subset K\left(Z_{s}, 1\right)$ with $f(x) \in \bigvee_{i=1}^{n} S_{i}^{1}$. For $s=1$ (that is, $R$ not a proper power, $R=Q$ ), $D(P)$ is exactly the cell-complex $C(P)$ described in the first section with $n 1$-cells and 1 2-cell. For $s>1, D(P)$ is an infinite dimensional cell-complex, with $n$-cells and $1 i$-cell for each $i \geqq 2$. By the Siefert-van Kampen Theorem, $\pi_{1}(D(P))$ is the pushout of the diagram

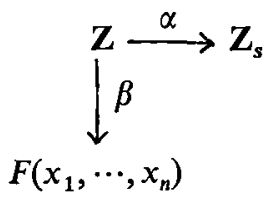


where $\alpha$ is an epimorphism and $\beta(t)=Q$. Thus, the pushout is the quotient of $F\left(x_{1}, \cdots, x_{n}\right)$ by the normal closure of the relation $Q^{s}=1$, that is, $G$.

TheOREM 2.1: Let $P$ be a one relator presentation of the group $G$. Then the cell-complex $D(P)$ is a $K(G, 1)$. In particular, if the relator is not a proper power, then the geometric dimension of $G$ is less than or equal to two.

Before giving our proof of this theorem, we observe several consequences of it and relations between it and previously known results.

If $P: a_{1}, b_{1}, \cdots, a_{g}, b_{g} ; \prod_{i=1}^{g}\left[a_{i}, b_{l}\right]$, then the relator is not a proper power and $C(P)$ is a compact, orientable surface of genus $g$. Theorem 2.1 asserts the asphericity of the surface. This familiar fact is thus proved in an unfamiliar manner. Similarly, this argument applies to the non-orientable surfaces except for the projective plane - for it the relator is a proper power.

CoRollary 2.2: If in the presentation $P$ of $G, R$ is not a proper power, and $N$ denotes the normal closure in $F\left(x_{1}, \cdots, x_{n}\right)$ of $R$, then there is an isomorphism $\psi: \mathbf{Z}(G) \rightarrow N /[N, N]$ of $\mathbf{Z}(G)$-modules with $\psi(1)$ the coset containing $R$.

(This is a special case of the Lyndon Identity Theorem [6].)

Proof: Let $X=C(P)$ and $X^{1}$ be its 1-skeleton $\left(=\bigvee_{i=1}^{n} S_{i}^{1}\right)$; let $\tilde{X} \rightarrow X$ be the universal covering of $X$ and $p^{1}: Y \rightarrow X^{1}$ be the restriction of $p$ to the 1-skeleta. We have the diagram

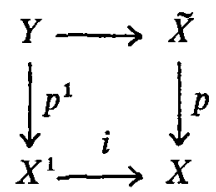

with $p^{1}$ and $p$ both covering maps. Any two points of $Y$ are end points of a path $w$ in $\tilde{X} ; p w$ is homotopic relative to its end points to a path $\hat{w}$ in $X^{1}$. Covering this homotopy in $\tilde{X}$ yields a path in $Y$ with the same end points as $w$; thus, $Y$ is patwise connected. A loop in $X^{1}$ which is null-homotopic in $X$ can be lifted to a loop in $Y$. Thus,

$$
p_{*}^{1} \pi_{1}(Y)=\operatorname{Kernel}\left(i_{*}: \pi_{1}\left(X^{1}\right)=F\left(x_{1}, \cdots, x_{n}\right) \rightarrow \pi_{1}(X)=F / N\right)=N .
$$

Since $p_{*}^{1}$ is an injection, $\pi_{1}(Y) \cong N$. By the Hurewicz Theorem $H_{1}(Y ; Z)$ $\cong N /[N, N]$. Since $X$ is aspherical, $\tilde{X}$ is both simply connected and aspherical. Thus, $\bar{X}$ is contractible. This implies it is acyclic. Thus,

$$
H_{2}(\tilde{X}, Y) \stackrel{\partial}{\cong} H_{1}(Y)
$$

But $Y$ is the 1-skeleton of $\tilde{X}$; and so, $H_{2}(\tilde{X}, Y)$ is the free abelian group on the 2-cells of $\hat{X}$. These are permuted simply transitively by $G$, the group of deck 
transformations. Hence, $H_{2}(\bar{X}, Y) \cong \mathbf{Z}(G)$ as $G$-modules; and $\mathbf{Z}(G) \cong N /[N, N]$ as $G$-modules.

It remains to describe the image of a generator. Let $e^{2}$ be the 2-cell containing the base point of $Y . \partial\left(e^{2}\right)$ is the homology class represented by the loop on the boundary. But $p_{*}^{1}$ of this loop is the class of the attaching map $R$.

Note: In this case the argument can be reversed; Lyndon's Identity Theorem implies that $\partial$ is an isomorphism and hence that $H_{2}(\tilde{X})=0$. Since $\widetilde{X}$ is simply connected and 2-dimensional, this implies it is contractible and $C(P)$ is aspherical. A similar argument was made by Cockcoft in [2].

COROLLARY 2.3: The group $G$ with a one relator presentation $P:\left\{x_{1}, \cdots, x_{n} ; R\right\}$ is torsion free if $R$ is not a proper power. If $R=Q^{s}, s>1$ and maximal, the order of $Q$ in $G$ is $s$.

This is a special case of results of Karrass, Magnus and Solitar [5].

Proof: If $R$ is not a proper power, by Theorem $2.1 C(P)$ is a $K(G, 1)$; and so, $c d(G) \leqq 2$. If $H$ is a non-trivial 1-generator subgroup of $G$, since $c d(H) \leqq c d(G)$, $H$ is not finite; i.e., $G$ is torsion free.

If $R=Q^{s}, s>1$ and maximal, since $D(P)$ has no cells out of $K\left(\mathbf{Z}_{s}, 1\right)$ in dimensions greater than two and the boundary of the 3-cell of $K\left(\mathbf{Z}_{s}, 1\right)$ lies in the 2-cell of $K\left(\mathbf{Z}_{s}, 1\right)$ it follows from standard computations of $H_{*}\left(\mathbf{Z}_{s} ; \mathbf{Z}\right)$, that $H_{3}(D(P) ; \mathbf{Z}) \cong \mathbf{Z}_{s}$. For a presentation $P^{\prime}:\left\{x_{1}, \cdots, x_{n} ; Q^{t}\right\}$, we have that $H_{3}\left(D\left(P^{\prime}\right) ; \mathbf{Z}\right) \cong \mathbf{Z}_{t}$. If $Q^{t}$ is trivial in $G$, and $t$ is minimal, it lies in the normal closure of $R$; by minimality, $R$ lies in the normal closure of $Q^{t}$. Thus, $P$ and $P^{\prime}$ present isomorphic groups, which have isomorphic homology groups. But this implies $t=s$.

There are some simple relations among the homology (and cohomology) of one relator groups immediately derivable from the geometry in Theorem 2.1.

Suppose $P:\left\{x_{1}, \cdots, x_{n} ; R\right\}$ is a one relator presentation of $G$ and $R=Q^{s}$, $\mathrm{s}>1$ and maximal. Then $P^{\prime}:\left\{x_{1}, \cdots, x_{n} ; Q\right\}$ is a one relator presentation of a group $G^{\prime}$. Let $\theta: G \rightarrow G^{\prime}$ be the surjection induced by the identity on generators.

COROLlary 2.4: For $M$ a left $G^{\prime}$-module, there is an exact sequence

$$
\begin{aligned}
0 & \rightarrow H_{2}\left(\mathbf{Z}_{s} ; M\right) \rightarrow H_{2}(G ; M) \stackrel{\theta_{*}}{\rightarrow} H_{2}\left(G^{\prime} ; M\right) \\
& \rightarrow H_{1}\left(\mathbf{Z}_{s} ; M\right) \rightarrow H_{1}(G ; M) \stackrel{\theta_{*}}{\rightarrow} H_{1}\left(G^{\prime} ; M\right) \\
& \rightarrow H_{0}\left(\mathbf{Z}_{s} ; M\right) \rightarrow H_{0}(G ; M) \stackrel{\theta_{*}}{\rightarrow} H_{0}\left(G^{\prime} ; M\right) \rightarrow 0 .
\end{aligned}
$$

If $S$ is the kernel of $\theta$, then for $q \geqq 3$ and any left $G$-nodule $L$, there is an isomorphism

$$
H_{q}\left(\mathbf{Z}_{s} ; L\right) \otimes \mathbf{Z}\left(G^{\prime}\right) \stackrel{\cong}{\longrightarrow} H_{q}(S ; L)
$$

of left $G^{\prime}$-modules. 
Proof: We modify the construction of $D(P)$ slightly. Instead of identifying the $S^{1}$ of $K\left(\mathbf{Z}_{s}, 1\right)$ with its image under the map into $\bigvee_{i=1}^{n} S_{i}^{1}$ spelling $Q$, first attach to $S^{1}$ the cylinder $S^{1} \times I$ along $S^{1} \times(0)$ and then identify $S^{1} \times 1$ into $\bigvee_{i=1}^{n} S_{i}^{1}$ to spell $Q$. Call this space $D^{\prime}(P)$; it obviously has the same homotopy type as $D(P)$. The advantage of $D^{\prime}(P)$ is that $K\left(\mathbf{Z}_{s}, 1\right)$ is a subspace of $\left(D^{\prime} P\right)$ (in fact it is imbedded as a cofibration). Let $C$ denote the cone of $K\left(\mathbf{Z}_{s}, 1\right)$. Then $C$ is contained in $C \cup D^{\prime}(P)$ as a cofibration and is contractible in itself to a point. Thus, $C \cup D^{\prime}(P)$ and $C \cup D^{\prime}(P) / C$ have the same homotopy type. But $C \cup D^{\prime}(P) / C$ is homeomorphic to $D\left(P^{\prime}\right)=C\left(P^{\prime}\right)$. Hence, we have the inclusions

$$
K\left(\mathbf{Z}_{s}, 1\right)<D^{\prime}(P)<C \cup D^{\prime}(P),
$$

where $D^{\prime}(P)$ is a $K(G, 1)$ and $C \cup D^{\prime}(P)$ is a $K\left(G^{\prime}, 1\right)$. Since

$$
\begin{aligned}
& H_{i}\left(D^{\prime}(P), K\left(\mathrm{Z}_{s}, 1\right) ; M\right) \\
& \quad \cong H_{i}\left(C \cup D^{\prime}(P), C ; M\right) \quad \text { (by excision) } \\
& \cong H_{i}\left(C\left(P^{\prime}\right) ; M\right) \\
& \cong H_{i}\left(G^{\prime} ; M\right),
\end{aligned}
$$

the exact sequence of the corollary is just the exact sequence of the pair $\left(D^{\prime}(P)\right.$, $\left.K\left(\mathbf{Z}_{s}, 1\right)\right)$. It is trivial on the left as indicated since $G^{\prime}$ has geometric dimension $\leqq 2$.

Let us abbreviate $K\left(\mathbf{Z}_{s}, 1\right)$ by $K$ and $D^{\prime}(P)$ by $X$. Let $X_{1} \rightarrow X$ be the regular covering of $X$ (a $K(G, 1))$ corresponding to the subgroup $S$ of $G$. Since $X$ is aspherical, so is $X_{1}$; hence, $X_{1}$ is a $K(S, 1)$. The group of deck transformations of the covering $X_{1} \rightarrow X$ is the quotient group $G^{\prime}$. This gives $H_{q}\left(X_{1} ; L\right)$ the structure of a $G^{\prime}$-module.

Since $\pi_{1}(K) \rightarrow \pi_{1}(X)$ factors through $\pi_{1}\left(X_{1}\right) \rightarrow \pi_{1}(X)$, the inclusion $K \rightarrow X$ factors through $X_{1} \rightarrow X$. The inverse $\hat{K}$ of $K$ in $X_{1}$ is then the disjoint union of copies of $K$, which are simply transitively permuted by the group $G^{\prime}$ of desk transformations. Hence,

$$
H_{q}(\hat{K} ; L) \cong H_{r}(K ; L) \otimes \mathbf{Z}\left(G^{\prime}\right)
$$

for all $q$. However, $\operatorname{dim} X-K=2$; and so, $\operatorname{dim} X_{1}-R=2$. Thus,

$$
H_{q}(\hat{K} ; L) \cong H_{q}\left(X_{1} ; L\right) \text { for } q \geqq 3 .
$$

Combining these two isomorphisms and the in.ormation that $X_{1}$ is a $K(S, 1)$ gives the conclusion

$$
H_{i}\left(\mathbf{Z}_{s} ; L\right) \otimes \mathbf{Z}\left(G^{\prime}\right) \cong H_{i}(S ; L) \text { for } q \geqq 3 .
$$

(Using results of Cohen and Lyndon [1], it can be shown that $S \cong *_{n^{\prime} \in G^{\prime}}\left(\mathbf{Z}_{\mathrm{s}}\right)_{g^{\prime}}$ with the obvious $G^{\prime}$ action.) 
We conclude this list of implications of Theorem 2.1 with an alternate proof of the Lyndon Identity Theorem

$P:\left\{x_{1}, \cdots, x_{n} ; R\right\}, R=Q^{s}, N=$ normal closure of $R$ in $F=F\left(x_{1}, \cdots, x_{n}\right)$ is a presentation of $G$ as before. The image of $Q$ in $G$ generates a cyclic subgroup $H$ of $G$ of order $s$.

COROLlary 2.5. (The Lyndon Identity Theorem): There is an isomorphism

$$
N /[N, N] \cong \mathbf{Z}[\mathrm{G} / H]
$$

of $G$-modules, where $\mathbf{Z}[G / H]$ is the free abelian group on the set of cosets $G / H$ of $H$ in $G$ with obvious $G$-action. It can be arranged so that the coset $H$ corresponds to the coset of $R$.

Proof: Let $p: X \rightarrow D(P)$ be the universal covering space of $D(P) . X$ has a cell-complex structure induced from that of $D(P)$ : the cells of $X$ lying over a single cell of $D(P)$ are permuted simply transitively among themselves by the group $G$ of deck transformations. Since $H_{k}\left(X^{(k)}, X^{(k-1)}\right)$ is the free abelian group on generators corresponding to the $k$-cells of $X$ (thus, the cells of $X^{(k)}-X^{(k-1)}$ ), it is thus a free $\mathbf{Z}(G)$-module with generators corresponding to the cells of $D(P)$.

Since $X$ is contractible, its homology is trivial; also, it is easy to verify that

$$
H_{i}(X)=H_{i}\left(X^{(k)}\right) \text { for } i<k .
$$

Thus, there are exact sequences

$$
\begin{aligned}
& 0 \rightarrow H_{2}\left(X^{(2)}\right) \rightarrow H_{2}\left(X^{(2)}, X^{(1)}\right) \rightarrow H_{1}\left(X^{(1)}\right) \rightarrow 0 \\
& 0 \rightarrow H_{3}\left(X^{(3)}\right) \rightarrow H_{3}\left(X^{(3)}, X^{(2)}\right) \rightarrow H_{2}\left(X^{(2)}\right) \rightarrow 0,
\end{aligned}
$$

etc. Splicing these together gives an exact sequence

$$
\cdots \rightarrow H_{4}\left(X^{(4)}, X^{(3)}\right) \rightarrow H_{3}\left(X^{(3)}, X^{(2)}\right) \stackrel{\partial_{3}}{\rightarrow} H_{2}\left(X^{(2)}, X^{(1)}\right) \stackrel{\partial_{2}}{\rightarrow} H_{1}\left(X^{(11}\right) \rightarrow 0
$$

of $\mathbf{Z}(G)$-modules.

We can assume $s>1$. (Corollary 2.2 established the conclusion for $s=1$.) We also assume the $K\left(\mathbf{Z}_{s}, 1\right)$ used to construct $D(P)$ has only one cell in each dimension and that the attaching map

$$
f: S^{1} \rightarrow \bigvee_{i=1}^{n} S_{i}^{1}
$$

is cellular. As in Corollary 2.2, $H_{1}\left(X^{(1)}\right) \cong N /[N, N]$. Thus cokernel $\partial_{3}$ $\cong N /[N, N]$. As in Corollary 2.2, the 2-cell $e^{2}$ containing the base point of $X$ can be taken as the generator for $H_{2}\left(X^{(2)}, X^{(1)}\right)$ and $\partial_{2} e_{2}$ is the coset of $R$ in $N /[N, N]$.

It remains only to describe $\partial_{3}$. Let $K=K\left(\mathbf{Z}_{s}, 1\right)$ and $q: \widetilde{K} \rightarrow K$ be the universal covering map. There is a map $\tilde{i}: \widetilde{K} \rightarrow X$ so that the diagram 


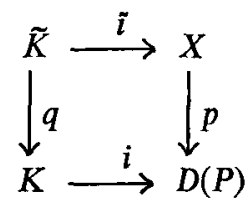

commutes. Let $e_{2}^{\prime}$ and $e_{3}^{\prime}$ be the 2- and 3-cells of $\tilde{K}$ containing the base point. It is a standard computation (p. 67 of [12]) that for

$$
\partial_{3}: H_{3}\left(\widetilde{K}^{3}, \widetilde{K}^{2}\right) \rightarrow H_{2}\left(\widetilde{K}^{2}, \widetilde{K}^{1}\right),
$$

$\partial_{3} e_{3}^{\prime}=(1-\mu) e_{2}^{\prime}$, where $\mu$ is the generator of $\pi_{1}(K)=H$. Thus,

$$
\partial_{3} i e_{3}^{\prime}=(1-\mu) \tilde{i} e_{2}^{\prime} \text {. }
$$

As $K$ contains all of the cells of $D(P)$ of dimensions 2 and greater, $\tilde{i} e_{3}^{\prime}=e_{3}$ and $i e_{2}^{\prime}=e_{2}$. Thus, the $\mathbf{Z}(G)$-homomorphism $\partial_{3}$ involves only the $\mathbf{Z}(H)$-module structure. Since $\mathbf{Z}(G)$ is a direct sum of copies of $\mathbf{Z}(H)$, one for each element of $G / H$, it is clear that cokernel $\partial_{3} \cong \mathbf{Z}[G / H]$.

\section{Proof of Theorem 2.1.}

The proof of Theorem 2.1 is inductive on $k$, the length of the word $Q$. The method we use to analyze a one-relator presentation goes back to Magnus [7].

Let us first briefly restate the construction. A group $G$ is given by a onerelator presentation $P:\left\{x_{1}, \cdots, x_{n} ; R\right\}$ with $R=Q^{s}, s$ maximal, $R$ being fully reduced. To the wedge of $n$ circles with base point is attached the circle with base point of a $K\left(\mathbf{Z}_{s}, 1\right)$ by a base point preserving map which "spells" the word $Q$. The resultant space is denoted $D(P)$. By the Seifert-van Kampen Theorem we know the fundamental group of $G$ to have the presentation $P$, and we need to verify only that $D(P)$ is aspherical to know that it is a $K(G, 1)$.

The case $s=1$, that is the case in which $R$ is not a proper power, can be read independently of the more general case. In this case, $D(P)=C(P)$ is two-dimensional, and certain complications, particularly in Sublemma 3.3.1, do not arise.

Being aspherical is an invariant of free homotopy type (homotopies not preserving the base point can be used) and altering the attaching map by a free homotopy does not change the free homotopy type of the space constructed. By using as attaching map a pointed map which "spells" a conjugate of $Q$, we are only altering by a free homotopy. Thus, to check $D(P)$ is aspherical, we can use $D\left(P^{\prime}\right)$ where

$$
R^{\prime}=\alpha R \alpha^{-1}=\left(\alpha Q x^{-1}\right)^{s} .
$$

In particular, we can assume $R$ and $Q$ are cyclically reduced.

LEMMA 3.1: If $Q$ has length $1, D(P)$ is aspherical.

Proof: Permuting the $x_{i}$ change $D(P)$ only by a homeomorphism. We will assume $Q=x_{1}^{ \pm 1}$ and $R=x_{1}^{ \pm s}$. The attaching map of the $S^{1}$ in $K\left(Z_{s}, 1\right)$ to 
$S_{1}^{1}$ can be taken to be a homeomorphism (it has degree \pm 1 as needed), and $D(P)=K\left(\mathrm{Z}_{s}, 1\right) \vee \bigvee_{i=2}^{n} S_{i}^{1}$, which by Theorem $W$ is aspherical.

We shall use the following notations: for $Q \in F\left(x_{1}, \cdots, x_{n}\right)$ and $a$ one of the $x_{i}$, $\#_{Q}(a)$ is the total number of occurences of $a$ in $Q$; i.e., the sum of the absolute values of the exponents of $a$ in $Q$, assuming $Q$ to be fully reduced; $\sigma_{Q}(a)$ is the exponent sum for $a$ in $Q$; i.e., the sum of the values of the exponents of $a$ in $Q$; the length of $Q, l(Q)=\sum_{i=1}^{n} \#_{Q}\left(x_{i}\right)$.

We assume that $k=l(Q) \geqq 2$ and that $D\left(P^{\prime}\right)$ is aspherical for any presentation $P^{\prime}$ for which $l\left(Q^{\prime}\right)<k$. This is our inductive hypothesis.

Lemma 3.2: Let $R=Q^{s}$, s maximal, be a cyclically reduced word in $F\left(x_{1}, \cdots, x_{n}\right)$. Suppose there is an $a=x_{I}$ for which

$$
\begin{aligned}
& 0 \neq \#_{Q}(a), 0=\sigma_{Q}(a) \text {, and } \\
& \sum_{i \neq I} \#_{Q}\left(x_{i}\right)<k .
\end{aligned}
$$

Then the inductive hypothesis implies that $D(P)$ is aspherical.

Corollary of Lemma 3.2: Let $R=Q^{s}$, s maximal, be a cyclically reduced word in $F\left(x_{1}, \cdots, x_{n}\right)$. If $Q$ has length $k$ and contains a generator with exponent sum 0 , then the inductive hypothesis implies $D(P)$ is aspherical.

LEMMA 3.3: Let $R=Q^{s}$, s maximal, be a cyclically reduced word in $F\left(x_{1}, \cdots, x_{n}\right)$. Suppose $Q$ has length $k$ and contains no generator with exponent sum 0 . Then the inductive hypothesis implies $D(P)$ is aspherical.

Proof of Lemma 3.2: Since $\sigma_{Q}(a)=0$, the function $F\left(x_{1}, \cdots, x_{n}\right) \rightarrow \mathbf{Z}$ defined by $W \rightarrow \sigma_{W}(a)$ induces a homomorphism of $G$ to the integers. Let $N$ be the kernel of this homomorphism. This proof will construct a regular covering space $X$ of $D(P)$ corresponding to the subgroup $N$ and prove $X$ is aspherical. The general structure of Lemma 1.1 will be reflected in these particular constructions. The construction of $X$ is motivated by the Reidemeister-Schreier rewriting process for $N$.

Let $\mathscr{B}=\left\{x_{i} \mid 1 \leqq i \leqq n, i \neq I\right\}$ and $W(\mathscr{B})$ be the wedge of $n-1$ circles labelled by the $x_{i}$ in $\mathscr{B}$. For each integer $i$ let $W(\mathscr{B})_{i}$ be a copy of the space $W(\mathscr{B})$. Let $T$ be the space obtained by attaching to the real line at each integer point $i$ the space $W(\mathscr{B})_{i}$ at its base point. For example, if $\mathscr{B}$ had two elements, part of $T$ would look like

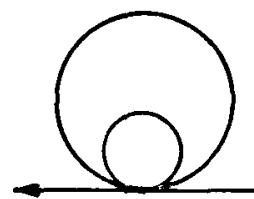

$-2$

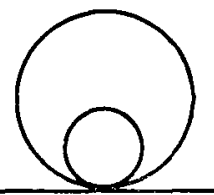

$-1$

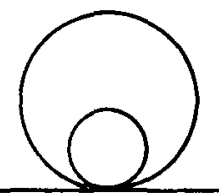

0

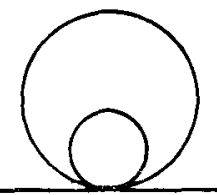

1

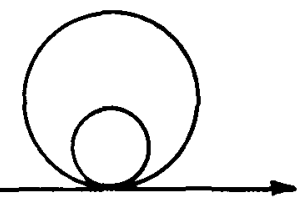

2 
Let $h: T \rightarrow T$ be the homeomorphism that carries a point of $W(\mathscr{B})_{i}$ into the corresponding point of $W(\mathscr{B})_{i+1}$ and takes a point $x$ of the real line to the point $x+1$. Clearly, $h$ generates an infinite cyclic group $H$ of self-homeomorphisms of $T$. The orbit space $T / H$ contains $W(\mathscr{B})$ (the image of any $W(\mathscr{B})_{i}$ ) and one more $S^{1}$ (the image of any interval $[k, k+1]$ of the real line for $k \in \mathbf{Z}$ ). Thus, $T / H$ can be identified with

$$
\bigvee_{i=1}^{n} S_{i}^{1} \equiv W(\mathscr{A})
$$

where $\mathscr{A}$ is the set $\left\{x_{1}, \cdots, x_{n}\right\}$, and

$$
p: T \rightarrow T / H=W(\mathscr{A})
$$

is a regular covering space.

It is easy to see that $\pi_{1}(T,(0))$ is free. A convenient set of generators are the $x_{\beta, i}$ described as follows: for $\beta \in \mathscr{B}, i \in \mathbf{Z}, x_{\beta, i}$ is the sum of the paths $[0, i]$, $S_{\beta}^{i} \in W(\mathscr{B})_{i}$, and $[i, 0]$. This is a loop at 0 . Clearly,

$$
p_{*}\left(x_{\beta, i}\right)=a^{i} \beta a^{-i} \in F\left(x_{1}, \cdots, x_{n}\right)=\pi_{1}(W(\mathscr{A}), *) .
$$

Let $S^{1}$ be the 1-sphere of $K\left(\mathbf{Z}_{s}, 1\right)$ and $f: S^{1} \rightarrow W(\mathscr{A})$ spell $Q$, i.e., $f_{*}(\mu)=Q$. Since $\sigma_{Q}(a)=0, f_{*}(\mu) \in \operatorname{Im}\left(p_{*}\right)$. Thus, there exists $f_{0}: S^{1} \rightarrow T$ (taking base point to $0 \in T$ ) such that $p f_{0}=f$.

Let $Q^{\prime}=f_{0 *}(\mu) \in \pi_{1}(T,(0))$. Recall that $\pi_{1}(T,(0))$ is free on the $x_{\beta, i}$. We claim

(i) $Q^{\prime}$ is not a proper power,

(ii) length $Q^{\prime}<k$, and

(iii) $Q^{\prime}$ is cyclically reduced.

The first is easy since $p_{*}\left(Q^{\prime}\right)=Q$ and $Q$ is not a proper power. Let $N=$ length $Q^{\prime}$ and write

$$
Q^{\prime}=x_{\beta_{1}, i_{1}}^{\varepsilon_{1}} \cdot x_{\beta_{2}, i_{2}}^{\varepsilon_{2}} \cdots \cdots \cdot x_{\beta_{N}, i_{N}}^{\varepsilon_{N}}
$$

$\varepsilon_{i}= \pm 1$. Then

$$
p_{*} Q^{\prime}=Q=\left(a^{i_{1}} \beta_{1}^{\varepsilon_{1}} a^{-i_{1}}\right)\left(a^{i} \beta_{2}^{\varepsilon_{2}} a^{-i_{1}}\right) \cdot \cdots \cdot\left(a^{i_{N}} \beta_{N}^{\varepsilon_{N}} a^{-i_{N}}\right) .
$$

Since $N=$ length $Q^{\prime}$, if $\left(\beta_{j}, i_{j}\right)=\left(\beta_{j+1}, i_{j+1}\right)$, then $\varepsilon_{j}=\varepsilon_{j+1}$. Hence, the only cancellation taking place in this expression for $Q$ is cancellation among the $a$ 's. Thus, $N=\Sigma_{\beta \in \mathscr{B}} \#_{Q} \beta<k$.

If $Q^{\prime}$ were not cyclically reduced, then $\left(\beta_{1}, i_{1}\right)=\left(\beta_{N}, i_{N}\right)$ and $\varepsilon_{1}+\varepsilon_{N}=0$. Thus, $Q$ starts with $a^{i_{1}} \beta^{\varepsilon_{1}}$ and ends with $\left(a^{i_{1}} \beta_{1}^{\varepsilon_{1}}\right)^{-1}$; i.e., it is not cyclically reduced.

Let $u=\min \left\{i_{j}\right\}$ and $v=\max \left\{i_{j}\right\}$ for $i_{j}$ appearing in $Q^{\prime}$. Since we can alter $f_{0}$ by a pointed homotopy, we can assume

$$
f_{0}\left(S^{1}\right) \subset \mathbf{R} \cup \bigcup_{i \in[u, v]} W(\mathscr{B})_{i} .
$$


For each integer $i$ let $K_{i}$ be a copy of $K\left(\mathbf{Z}_{s}, 1\right)$ and $C_{i}$ be the 1-sphere of $K_{i}$. Let $X$ be the quotient space of the disjoint union of the $K_{i}$ 's and $T$ by identifying each point $P \in C_{i}$ with $h^{i}\left(f_{0}(P)\right) \in T$. If is clear that $h$ extends to a self-homeomorphism of $X$, which we also call $h$ (it takes a point of $K_{i}$ to the corresponding point of $K_{i+1}$ ). Again, $h$ generates an infinite cyclic group $H$ of self-homeomorphisms of $X$. It is clear that the orbit space $X / H$ is

$$
T / H \underset{p f_{0}}{\cup} K=\bigvee_{i=1}^{m} S_{\imath}^{1} \bigcup_{f} K=D(P) .
$$

Also, the action of $H$ on $X$ is properly discontinuous; and so,

$$
X \rightarrow X / H=D(P)
$$

is a covering map. Thus, it suffices to show that $X$ is aspherical.

Let $X_{N}$ be the subcomplex of $X$ consisting of the real line, the $W(\mathscr{B})_{i}$ for $u+N \leqq i \leqq v+N$ and $K_{N}$. Clearly, $h\left(X_{N}\right)=X_{N+1}$. Our inductive hypothesis will show $X_{N}$ is aspherical, and we will use this to conclude that $X$ is aspherical.

Sublemma 3.2.1: a) $X_{N}$ is aspherical,

b) the elements $x_{i, i}, \beta \in \mathscr{B}, i \in[u+N+1, v+N]$, are distinct and generate a free subgroup of $\pi_{1}\left(X_{N},(0)\right)$, and

c) the elements $x_{\beta}, \quad \beta \in \mathscr{B}, \quad i \in[u+N, v+N-1]$, are distinct and generate a free subgroup of $\pi_{1}\left(X_{N},(0)\right)$.

ProOF: It suffices to consider the case $N=0$. Since the real line is a contractible subcomplex of $X_{0}$, collapsing it to a point does not change homotopy type. Call the resultant space $Y_{0}$ and $q: X_{0} \rightarrow Y_{0}$ the collapsing map. Let $W$ be the subcomplex of $Y_{0}$ which is the image of all $W(\mathscr{B})_{i}, u \leqq i \leqq v$. This is a wedge of circles, indexed by $\mathscr{B} \times(u, u+1, \cdots, v)$. $Y_{0}$ is of the form $W \cup_{q f_{0}} K$, for $q f_{0}: C \rightarrow K$. Let $Q^{\prime}=\left(q f_{0}\right)_{*}(\mu) \in \pi_{1}(W)$. It is clear that $Y_{0}$ is $D\left(P^{\prime}\right)$ for the presentation $P^{\prime}:\left\{\mathscr{B} \times(u, u+1, \cdots, v) ; Q^{\prime s}\right\}$, where $Q^{\prime}$ is as above. The inductive hypothesis then implies that $Y_{0}$, and hence $X_{0}$, is aspherical.

The definition of $u$ is such that $b$ ) is an immediate consequence of the Magnus Freeness Theorem. Similarly, c) follows from the definition of $v$.

Sublemma 3.2.2: For $X_{0, N}=X_{0} \cup X_{1} \cup \cdots \cup X_{N}, X_{0, N}$ is aspherical and $X_{N} \rightarrow X_{0, N}$ induces and injection of fundamental groups.

Proof: The assertion is true for $N=0$ by Sublemma 3.2.1. Assume it is true for $N$. Then $X_{0, N+1}=X_{0, N} \cup X_{N+1}$ and

$$
X_{0, N} \cap X_{N+1}=(\text { real line }) \cup W(B)_{u+N+1} \cup \cdots \cup W(B)_{v+N} .
$$

By the Siefert-van Kampen Theorem, the following diagram is a pushout in the category of groups 


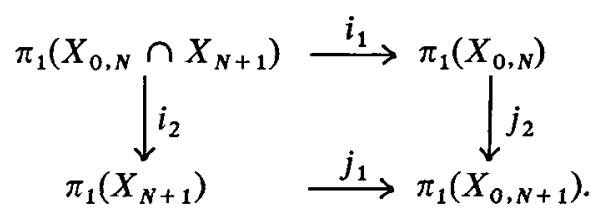

Sublemma 3.2.1.c) shows that $i_{2}$ is an injection. The map $i_{1}$ factors through $\pi_{1}\left(X_{N}\right)$ and is thus the composition of two injections, by sublemma 3.2.1.b) and induction. Hence, $\pi_{1}\left(X_{0 N+1}\right)$ is a free product with amalgamation, and, in particular, $j_{1}$ is an injection. The conclusion that $X_{0, N+1}$ is aspherical then follows from Theorem $W$.

We now conclude the proof of Lemma 3.2 by showing $X$ to be aspherical. Let $F: S^{q} \rightarrow X$ be a continuous map. Since $S^{q}$ is compact, there is an integer $N$ such that $F\left(S^{q}\right) \subset X_{-N} \cup X_{-N+1} \cup \ldots \cup X_{N}$, a subspace homeomorphic to $X_{0,2 N}$. Since $X_{0,2 N}$ is aspherical, $F$ is null-homotopic if $q \geqq 2$. Thus, $X$ is aspherical.

Proof of Lemma 3.3: Since $s$ is maximal and $k \geqq 2, Q$ must involve at least two generators. Let $a$ and $b$ be two such and $\mathscr{C}$ be set of all other $x_{i}, 1 \leqq i$ $\leqq n$. Let $A$ and $B$ be two objects distinct from any in $F\left(X_{1}, \cdots, x_{n}\right)$ and $G$. Let $\mathscr{A}^{\prime}=\mathscr{C} \cup\{A\} \cup\{b\}$ and $\mathscr{A}^{\prime \prime}=\mathscr{C} \cup[A] \cup[B]$ and let $F\left(\mathscr{A}^{\prime}\right)$ and $F\left(\mathscr{A}^{\prime \prime}\right)$ be the free groups generated by the elements of $\mathscr{A}^{\prime}$ and $\mathscr{A}^{\prime \prime}$, respectively. Define homomorphisms

and

$$
\theta_{1}: F\left(x_{1}, \cdots, x_{n}\right) \rightarrow F\left(\mathscr{A}^{\prime}\right)
$$

$$
\theta_{2}: F\left(\mathscr{A}^{\prime}\right) \rightarrow F\left(\mathscr{A}^{\prime \prime}\right)
$$

by $\theta_{1}(a)=A^{q}, \theta_{1}(b)=b, \theta_{1}(c)=c$ for all $c \in \mathscr{C}$, and $\theta_{2}(A)=A, \theta_{2}(b)=B A^{-p}$, $\theta_{2}(c)=c$ for all $c \in \mathscr{C}$, where $q=\sigma_{Q}(b)$ and $p=\sigma_{Q}(a)$. Let $Q^{\prime}=\theta_{1}(Q) \in F\left(\mathscr{A}^{\prime}\right)$ and $Q^{\prime \prime}=\theta_{2}\left(Q^{\prime}\right) \in F\left(. \mathscr{A}^{\prime \prime}\right)$. It is immediate that $Q^{\prime}$ and $Q^{\prime \prime}$ are cyclically reduced and are not proper powers. Also,

and

$$
\begin{aligned}
\sigma_{Q^{\prime \prime}}(A) & =\sigma_{Q^{\prime}}(A)-p \sigma_{Q^{\prime}}(b) \\
& =q \sigma_{Q}(a)-p \sigma_{Q^{\prime}}(b) \\
& =0,
\end{aligned}
$$

$$
\#_{Q^{n}} B=\#_{Q^{b}} \text { and } \#_{Q^{\prime \prime}} c=\#_{Q} c \text { for all } c \in \mathscr{C} \text {. }
$$

Lemma 3.2 applies to the presentation

$$
P^{\prime \prime}=\left\{\mathscr{A}^{\prime \prime} ; Q^{\prime \prime s}\right\} ;
$$

i.e., $D\left(P^{\prime \prime}\right)$ is aspherical. Let $P^{\prime}$ be the presentation $\left\{\mathscr{A}^{\prime}, Q^{\prime s}\right\}$. 
Sublemma 3.3.1. $D\left(P^{\prime}\right)$ and $D\left(P^{\prime \prime}\right)$ have the same homotopy type.

Proof of Sublemma 3.3.1: Let $S^{1} \subset K\left(\mathbf{Z}_{s}, 1\right)$ and

$$
f^{\prime}: S^{1} \rightarrow S_{A}^{1} \vee S_{b}^{1} \vee \underset{c \in \mathscr{C}}{\vee} S_{c}^{1}
$$

be a map "spelling" $Q$ '. Let

$$
h: S_{A}^{1} \vee S_{b}^{1} \vee \underset{c \in \mathscr{C}}{\bigvee} S_{c}^{1} \rightarrow S_{A}^{1} \bigvee S_{B}^{1} \bigvee \underset{c \in \mathscr{C}}{\bigvee} S_{c}^{1}
$$

induce the homomorphism $\theta_{2}$ on fundamental groups. Then $h f^{\prime}$ "spells" $Q$ ". Since $\theta_{2}$ is an isomorphism, $h$ is a homotopy equivalence; and so $D\left(P^{\prime}\right)$ and $D\left(P^{\prime \prime}\right)$ are homotopy equivalent.

We next compare $D(P)$ and $D\left(P^{\prime}\right)$ in order to deduce the asphericity of $D(P)$. Let $M$ be the mapping cylinder of a map from $S^{1}$ to $S^{1}$ of degree $q$. Recall that $M$ has in it circles $S_{d}^{1}$ and $S_{r}^{1}$ (domain and range) such that $S_{r}^{1}$ is a strong deformation retract of $M$, and the inclusion of $S_{d}^{1}$ in $M$ followed by the retraction of $M$ onto $S_{r}^{1}$ is a map of degree $q$. Let $\bar{W}=M \vee S_{b}^{1} \vee V_{c \in \mathscr{C}} S_{c}^{1}$; then $W(\mathscr{A})$ $=S_{d}^{1} \vee S_{b}^{1} \vee \bigvee_{c \in \mathscr{C}} S_{c}^{1}$ and $W\left(\mathscr{A}^{\prime}\right)=S_{r}^{1} \vee S_{b}^{1} \vee \bigvee_{c \in \mathscr{C}} S_{c}^{1}$ are contained in $\vec{W}$. Clearly, $W\left(\mathscr{A}^{\prime}\right)$ is a strong deformation retract of $\bar{W}$ and the composition of $i: W(\mathscr{A}) \rightarrow \bar{W}$ followed by the retraction of $\bar{W}$ onto $W\left(\mathscr{A}^{\prime}\right)$ is a map $k: W(\mathscr{A})$ $\rightarrow W\left(\mathscr{A}^{\prime}\right)$ which induces the homomorphism $\theta_{1}: F(\mathscr{A}) \rightarrow F\left(\mathscr{A}^{\prime}\right)$ on fundamental groups.

Let $f: S^{1} \rightarrow W(\mathscr{A})$ spell $Q$. Then $D(P)=W(\mathscr{A}) \cup_{f} K$ is a subcomplex of $X=W \cup_{i o f} K$, where $S^{1} \subset K=K\left(Z_{s}, 1\right)$. Let $r: \bar{W} \rightarrow W\left(. \mathscr{A}^{\prime}\right)$ be the retraction. Then $W\left(\mathscr{A}^{\prime}\right) \cup_{\text {ro(if) }} K$ has the same homotopy type as $X$. (This uses the general proposition that if $W \rightarrow D$ is a cofibration and $k: W \rightarrow W^{\prime}$, then $D \cup_{k} W^{\prime}$ and $D \cup M(k)$ have the same homotopy type. In our case, $M(k)=\bar{W}$.) The map $r \circ i \circ f: S^{1} \rightarrow W\left(\mathscr{A}^{\prime}\right)$ spells $Q^{\prime}$. Thus, $W\left(\mathscr{A}^{\prime}\right) \cup_{\text {ro(if })} K$ is $D\left(P^{\prime}\right)$. Since this is aspherical, so is $X$.

Let $X_{1}$ be that part of $X$ which is in $M$ and has cylinder coordinate $\geqq \frac{1}{2}$. Let $X_{2}$ be the closure in $X$ of the complement of $X_{1}$. Then $X_{1}$ has $S_{r}^{1}$ as strong deformation retract, $X_{2}$ has $D(P)$ as strong deformation retract, and $X_{0}=X_{1}$ $\cap X_{2}$ is a circle.

By the Siefert-van Kampen Theorem, the following is a pushout diagram in the category of groups:

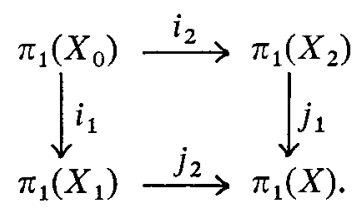

From remarks above, the groups on the left are both infinite cyclic and $i_{1}$ is raising to the $q$ th power. Since $X_{2}$ has the homotopy type of $D(P), \pi_{1}\left(X_{2}\right)=G$, the 
group presented by $P$. By construction $i_{2}$ takes a generator into the element of $G$ represented by $a$. By the Freeness Theorem (recall that $R$ is cyclically reduced and involves $b) i_{2}$ is an injection. Thus, $\pi_{1}(X)$ is a free product with amalgamation and $j_{1}$ and $j_{2}$ are injections.

Consider the universal covering $p: \tilde{X} \rightarrow X$ of $X$. Since $X$ is aspherical, $\tilde{X}$ is contractible, and has trivial homology groups. Thus, by the Mayer-Vietoris Theorem

$$
H_{i}\left(p^{-1} X_{0}\right) \rightarrow H_{i}\left(p^{-1} X_{1}\right) \oplus H_{i}\left(p^{-1} X_{2}\right)
$$

is an isomorphism for $i \geqq 1$. For $\alpha=0,1,2, \pi_{1}\left(X_{\alpha}\right) \rightarrow \pi_{1}(X)$ is an injection; hence each component of $p^{-1} X_{\alpha}$ is homomorphic to the universal covering space of $X_{\alpha}$. In particular, $X_{0}$ being a circle, each component of $p^{-1} X_{0}$ is the real line, and $H_{i}\left(p^{-1} X_{0}\right)=0$ for $i \geqq 1$. Thus, $H_{i}\left(p^{-1} X_{2}\right)=0$ for $i \geqq 1$; i.e., the universal covering space of $X_{2}$ is acyclic. Since it is simply connected, it is contractible. Hence, $X_{2}$ is aspherical, and so is $D(P)$.

\section{An amusing space}

In [4] Higman presented a group $\mathbf{H}$ on four generators and four relators which has a number of interesting properties. We observe here that the canonical two-dimensional cell-complex of his presentation is an aspherical space and translate various of the group -theoretic properties of $\mathbf{H}$ into topological properties of the associated cell-complex.

Let $H_{x y}$ be the group presented by $P_{x, y}:\left\{x, y ; x y x^{-1} y^{-2}\right\}$. Since $x y x^{-1} y^{-2}$ has exponent sum equal -1 , it is not a proper power and so by Theorem 2.1, $C\left(P_{x y}\right)$ is aspherical. Let $H_{x y z}$ be the free product with amalgamation defined by

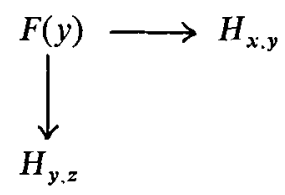

where each homomorphism carries $y$ into its class in the appropriate group. By Theorem $W, C\left(P_{x, y}\right) \cup_{y} C\left(P_{y . z}\right)$ is aspherical. As seen in [4], the homomorphism

$$
F(x, z) \rightarrow H_{x, y, z},
$$

defined by taking each of $x$ and $z$ into its class in $H_{x y . z}$, is an injection. Thus letting $\mathbf{H}$ be the free product with amalgamation defined by

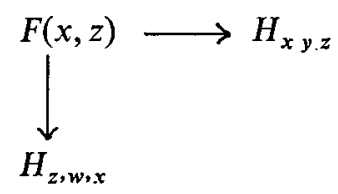


the space $\left(C\left(P_{x, y}\right) \bigcup_{y} C\left(P_{y, z}\right)\right) \bigcup_{x, z}\left(C\left(P_{z, w}\right) \bigcup_{w} C\left(P_{w, x}\right)\right)$ is a $K(\mathbf{H}, 1)$. This space is $C(P)$ for the presentation

$$
P:\left\{w, x, y, z ; w x w^{-1} x^{-2}, x y x^{-1} y^{-2}, y z y^{-1} z^{-2}, z w z^{-1} w^{-2}\right\}
$$

of $H$. It has one vertex, four 1-cells and four 2-cells; it is aspherical and $\pi_{1}(C(P), *)$ $\cong \mathbf{H}$. Since the abelianization of $\mathbf{H}$ is trivial, $H_{1}(C(P) ; \mathbf{Z})=0$. Since the boundary of a non-zero 2-chain corresponding to the cell attached by the relation in $P_{x, y}$ is a non-zero multiple of the 1-chain corresponding to $y$, it is easy to see that the only 2-cycle on $C(P)$ is the 0 cycle. Thus, $H_{2}(C(P) ; Z)=0$. Clearly the higher dimensional homology groups of $C(P)$ are all zero. This implies that the suspension of $C(P)$ is contractible.

The group $\mathbf{H}$ has no non-trivial finite dimensional representations over any field, see [4]. It follows that if $\mathscr{B}$ is any local coefficient system over $C(P)$ of finitely generated abelian groups, then

$$
\tilde{H}_{*}(C(P) ; \mathscr{B})=0=\tilde{H}^{*}(C(P) ; \mathscr{B}) \text {. }
$$

Also, any finite dimensional real or complex vector bundle over $C(P)$ is trivial since obstructions to finding a section are elements of suitable cohomology groups.

Summarizing, $C(P)$ is a finite 2-dimensional cell-complex (nine cells) $C(P)$ is a $K(\mathbf{H}, 1)$, for $\mathbf{H}$ the Higman group, the suspension of $C(P)$ is contractible, homology and cohomology of $C(P)$ with finitely generated local coefficients is trivial, all finite dimensional real or complex vector bundles over $C(P)$ are trivial. In short, it is difficult to detect that $C(P)$ does not have the homotopy type of a point by the usual abelian invariants of algebraic topology. (If one performs the same construction on the analogous presentation, cycling around in three steps, instead of four, as here, the complex obtained does have the homotopy type of a point.)

If $N$ is a regular neighborhood of an imbedded $C(P)$ in $\mathbf{R}^{4}$ and $S$ is the union of two copies of $N$ identified along the boundary of $N$, the "double" of $N$, then $S$ is an orientable 4-manifold and is a homology 4-sphere. It can be shown that $\pi_{1}(S) \cong \mathbf{H}$. Except for infinitely generated local coefficient systems, the manifold $S$ is hard to distinguish from $S^{4}$ by usual invariants of algebraic topology.

\section{Conjecture $\mathbf{I}^{\prime}$}

In 1961 Papakyriakopoulos conjectured [8] that for the presentation

$$
\widetilde{P}:\left\{a, b, x_{1}, y_{1}, \cdots, x_{n}, y_{n} ;[a, b] \prod_{i=1}^{n}\left[x_{i}, y_{i}\right],[a, b \tau]\right\},
$$

where $\tau$ is in the commutator subgroup of the free group on $a, b, x_{1}, \cdots, y_{n}$, the complex $C(\widetilde{P})$ is aspherical.

In 1963 Rapaport showed [9] that for the presentation 


$$
P:\left\{a, b, x_{1}, y_{1}, \cdots, x_{n}, y_{n} ;[a, b] \prod_{i=1}^{n}\left[x_{i}, y_{i}\right],[a, w]\right\},
$$

where $w$ is any word in $a, b, \cdots, y_{n}$, the group defined by $P$ is torsion free. (This established a generalization of Conjecture I of Papakyriakopoulos; the statement above for $\widetilde{P}$ is his Conjecture $I^{\prime}$.)

We show here, using specific results in Rapaport's argument, that if $[a, w]$ is not in the normal closure in $F\left(a, b, x_{1}, \cdots, y_{n}\right)$ of the word $[a, b] \prod_{i=1}^{n}\left[x_{i}, y_{i}\right]$, then $C(P)$ is aspherical. Of course, if $[a, w]$ were in that normal closure, then $C(P)$ would be the homotopy type of the wedge of a surface of genus $n+1$ and a 2 sphere; thus, $C(P)$ would not be aspherical. The word $[a, b \tau]$ in Papakyriakopoulos Conjecture is not in the normal closure of $[a, b] \prod_{i=1}^{n}\left[x_{i}, y_{i}\right]$, (see Theorem 6.2 of [8]), and the above assertion then implies that Conjecture $I^{\prime}$ is true.

Before giving this argument, we would like to make two comments. There is a vast difference between asking whether the group defined by a presentation $P$ is torsion free and asking if $C(P)$ is aspherical. The latter conclusion implies the group has geometric dimension two, a rare property even among groups without torsion. G. Baumslag has given (unpublished) an example of an infinite dimensional, finitely presented group without torsion; it is known that for each integer $n$, there exists a finitely presented group with geometric dimension $n$. Of course, all of them are torsion free. Secondly, although our argument establishes Conjecture $I^{\prime}$, it is not in the geometric spirit sought by Papakyriakopoulos. Indeed, it relies heavily on the specific rewriting obtained by Rapaport. While this is a failing from the viewpoint of [8] and even though there may be some geometric argument applicable for $\tilde{P}$, we would like to point out that the combination of rewriting and covering space techniques employed here has a general character which may be applicable to a wider class of groups.

We first modify the presentation $P$ to obtain a new presentation $P^{\prime}$, differing from $P$ only in that a word $\left[w^{*}, a\right]$ with $\sigma_{w^{*}} a=0$ is substituted for the word $[a, w]$ in $P$. As we have seen $C(P)$ is aspherical if and only if the corresponding construction with the conjugate $w^{-1}\left(a w a^{-1} w^{-1}\right) w$ is aspherical (Lemma 1.2). Writing

$$
w^{-1} a w a^{-1}=w^{-1} a^{h} a a^{-h} w a^{-1},
$$

let $w^{*}=w^{-1} a^{h}$ for that $h$ with $\sigma_{w-1 u^{h}} a=0$; then $w^{-1} a w a^{-1}=\left[w^{*}, a\right]$ and $\sigma_{w^{*}} a=0$. Letting $n=g-1$ and writing

$$
\begin{aligned}
\chi_{2 i-1} & =x_{i} \\
\chi_{2 i} & =y_{i}, i=1, \cdots, g-1,
\end{aligned}
$$

we have precisely the presentation in [9].

Continuing with the notation and results of [9], let 


$$
\begin{aligned}
b_{k} & =a^{-k} b a^{k} \\
x_{i . k} & =a^{-k} \chi_{i} a^{k} \\
{[j j] } & =\prod_{i=1}^{g-1}\left[x_{2 i-1 j}, x_{2 i, j}\right] .
\end{aligned}
$$

The relator $G^{*}=[a, b] \cdot[00]$ becomes

$$
G^{*}=G_{0}=b_{-1} b_{0}^{-1}[00],
$$

and

Let

$$
G_{k}=a^{-k} G^{*} a^{k}=b_{k-1} b_{k}^{-1}[k k]
$$

and

$$
R=w^{*} a w^{*-1} a^{-1}=w_{0} w_{-1}^{-1} \text {, }
$$

$$
R_{k}=w_{k} w_{k-1}^{-1}
$$

where $R_{k}=a^{-k} R a^{k}$ and $w_{k}=a^{-k} w_{0} a^{k}$.

If $R$ is not in the normal closure of $G^{*}$, there are integers $(u, v)$ with either $u=v=0$ or $u \leqq 0$ and $1 \leqq v$ and a word $A=A_{0}$ in $F_{0}=F\left(x_{i \cdot k}, b\right.$ for $u \leqq k$ $\leqq v$ ) such that, letting

$$
\begin{aligned}
& H_{r}:\left\{F_{r} ; A_{r}\right\} \\
& S_{r}: F\left(x_{i k}, b_{r} \text { for } r+u+1 \leqq k \leqq r+v\right),
\end{aligned}
$$

$\left(=F\left(b_{r}\right)\right.$ if $u=v=0$ ),

$\alpha_{r}: S_{r} \rightarrow H_{r}$ be induced by the identity on generators,

$\beta_{r}: S_{r} \rightarrow H_{r+1}$ be induced by the identity on the $x_{t, k}$ and $\beta_{r}\left(b_{r}\right)=[r+1$ $r+1]^{-1} b_{r+1}$, then $\alpha_{r}$ and $\beta_{r}$ are injections,

$A_{r}$ is not a prope ${ }_{i}$ power in $F_{r}$,

$H=\cdots * H_{-1 S-1}^{*} H_{0} * s_{0} H_{1} * \cdots$ is the kernel of $G(P) \rightarrow \mathbf{Z}(a)$ defined by exponent sum of $a$,

$\mathbf{Z}(a)$ acts as group of automorphisms on $H$ with the generator $a$ translating the above displayed generalized free product one step to the right (by conjugation), and

the modified presentation $P^{\prime}$ of $G$ is presisely that defined by this extension.

As in Section 3 we will construct an aspherical, 2-dimensional call-complex for $H$ with a properly discontinuous action of $\mathbf{Z}(a)$ on it inducing the above automorphisms on $H$; the quotient space defined by this action will be $\left(C P^{\prime}\right)$. As it has an aspherical covering space, it will also be aspheric.l.

Let $X_{0}$ be the cell-complex constructed as follows: to the real line adjoin at each integer point $k, u \leqq k \leqq v$, a wedge of $2 g-2$ circles corresponding to the generators $x_{i, k}, i=1, \cdots, 2 g-2$, and one more circle at 0 corresponding to $b$. Let 0 be the base point and describe the generator in $\pi_{1}\left(X_{0}, 0\right)$ corresponding to $x_{i, k}$ by the sum $[0, k]+S_{i k}^{1}+[k, 0]$. Attach a 2 -cell to $X_{0}$, forming $Y_{0}$, to spell 
the word $A_{0}$ with the above description of $F_{0}$. As $A_{0}$ is not a proper power in $F_{0}$, $Y_{0}$ with the line identified to a point is $C\left(H_{0}\right)$ and is aspherical by Theorem 2.1 . Thus, $Y_{0}$ is aspherical.

For each integer $r$ let $Y_{r}$ be a copy of $Y_{0}$ translated $r$ to the right. Take all of the $Y_{r}$ to be disjoint. Attach the sylinder $\left(S^{1} \times I\right)_{r+1}$ to $Y_{r+1}$ along $S^{1} \times 1$ by a mapping $\beta_{r}$ which spells $[r+1 r+1]^{-1} b_{r+1}$. Since $Y_{r+1}$ is a strong deformation retract of $Y_{r+1} \bigcup_{B r}\left(S^{1} \times 1\right)_{r+1}=W_{r+1}$, the space $W_{r+1}$ is aspherical; it is a $K\left(H_{r+1}, 1\right)$. Let $Z_{r}$ be the complex consisting of the real line with circles corresponding to $x_{i k}, 1 \leqq i \leqq 2 g-2, r+u+1 \leqq k \leqq r+v$, adjoined at $k$, together with a circle corresponding to $b_{r}$ adjoined at $r$.

Define $a_{r}: Z_{r} \rightarrow W_{r}$ to be the identity on the line and to carry the circle in $Z_{r}$ corresponding to $x_{i, k}$ (to $b_{r}$ ) homeomorphically into the circle in $W_{r}$ corresponding to $x_{i . k}$ (to $b_{r}$ ). Define $\bar{\beta}_{r}: Z_{r} \rightarrow W_{r+1}$ to be the identity on the line, to carry the circle in $Z_{r}$ corresponding to $x_{i, k}$ homeomorphically into the circle in $W_{r+1}$ corresponding to $x_{i k}$, and to carry the circle corresponding to $b_{r}$ homeomorphically into the circle $\left(S^{1} \times 0\right)_{r+1}$ in $W_{r+1}$. Then $\bar{a}_{r}$ and $\bar{\beta}_{r}$ induce the homomorphisms $\alpha_{r}$ and $\beta_{r}$ on fundamental groups. As these are injections, by Theorem $W$, $W_{r} \bigcup_{z_{r}} W_{r+1}$ has fundamental group $H_{r}{ }_{S_{r}}^{*} H_{r+1}$ and is aspherical. Since $\pi_{1}\left(W_{t}\right)$ $\rightarrow \pi_{1}\left(W_{r} \cup_{Z_{r}} W_{r+1}\right), i=r, r+1$, is injective, so are the compositions

and

$$
\pi_{1}\left(Z_{r-1}\right) \stackrel{\beta_{r-1}}{\longrightarrow} \pi_{1}\left(W_{r}\right) \longrightarrow \pi_{1}\left(W_{r} \bigcup_{Z_{r}} W_{r+1}\right)
$$

$$
\pi_{1}\left(Z_{r+1}\right) \stackrel{\alpha_{r+1}}{\longrightarrow} \pi_{1}\left(W_{r+1}\right) \longrightarrow \pi_{1}\left(W_{r} \bigcup_{Z_{r}} W_{r+1}\right)
$$

Thus, continuing inductively, the process can be iterated to form' a space

$$
X=\cdots \cup W_{-1} \bigcup_{z_{-1}} W_{0} \bigcup_{z_{1}} W_{1} \cup \cdots
$$

which is a $K(H, 1)$. There is a properly discontinuous action of $\mathbf{Z}(a)$ defined on $X$ by letting $a$ move each point one unit to the right or into its correspondent with one higher subscript $r$ or $k$, as the case may be. The quotient space $X / Z(a)$ has

a base point, (0)

$$
\begin{array}{ll}
2 g-2 & \text { circles, }\left(x_{i k}\right), i=1, \cdots, 2 g-2 \\
1 & \text { circle, }\left(b_{k}\right) \\
1 & \text { circle, image }[0,1] \\
1 & \text { 2-cell, attached by } A_{0}, \text { and } \\
1 & \text { 2-cell, attached by } b_{1}=\left[\begin{array}{ll}
1 & 1
\end{array}\right] b_{0} .
\end{array}
$$

These correspond precisely to the generators and relators of the presentation $P^{\prime}$. 
Thus, $X / \mathbf{Z}(a)$ is homeomorphic to $C\left(P^{\prime}\right)$. Since $X$ is aspherical and the projection $X \rightarrow X / \mathbf{Z}(a)=C\left(P^{\prime}\right)$ is a covering map, $C\left(P^{\prime}\right)$ is aspherical.

\section{References}

[1] D. E. Cohen and R. C. Lyndon, 'Free bases for normal subgroups of free groups', Trans. Amer. Math. Soc. 108 (1963), 526-537.

[2] W. H. Coc'scroft, 'On two-dimensional aspherical complexes', Proc. London Math. Soc. 4 (1954), 375-384.

[3] S. Eilenberg and T. Ganea, 'On the Lustern:ck-Schnirelmann category of abstract groups', Ann. Math. 65 (1957), 517-518.

[4] G. Higman, 'A finitely generated infinite simple group', J London Math. Soc. 26 (1951), 61-64.

[5] A. Karrass, W. Magnus and D. Solitar, 'Elements of finite order in groups with a single defining relation', Comm. Pure and Appl. Math. 13 (1960), 57-66.

[6] R. C. Lyndon, 'Cohomology theory of groups with a single defining relation', Ann. Math. 52 (1950), 650-665.

[7] W. Magnus, 'Ủber diskontinuierliche Gruppen mit einer definierenden Relation. (Der Freiheitssatz)', J. reine angew. Math. 163 (1930), 141-165.

[8] C. D. Papakyriakopoulos, 'Attaching 2-dimensic nal cells to a complex', Ann. Math. 78 (1963), 205-222.,

[9] E.S. Rapaport, 'Proof of a conjecture of Papakyriakopoulos', Ann. Math. 79 (1964), 506-513.

[10] J. R. Stallings, 'A finitely presented group whose 3- dimensional integral homology' is not finitely presented', Amer. J. Math. 85 (1963), 541-543.

[11] J. R. Stallings, 'On torsion free groups with infinitely many ends', Ann. Math. 88 (1968), 312-334.

[12] N. E. Steenrod, Cohomology Operations, (Princeton Univ. Press, 1962).

[13] R. G. Swan, 'Groups of cohomological dimension one', J. Algebra, 12 (1969), 585-610.

[14] J. H. C. Whitehead, 'On the asphericity of regions in a 3-sphere', Fund. Math. 32 (1939), 149-166.

The Graduate School

City University of New York,

33 West 42 Street

New York, 10036

U. S. A. 